\title{
GENERATION OF FEMTOSECOND ELECTRON AND PHOTON PULSES
}

\author{
C. Thongbai *, V. Jinamoon, N. Kangrang, K. Kusoljariyakul, S. Rimjaem, J. Saisut, T. Vilaithong, \\ FNRF, Chiang Mai University, Chiangmai Thailand \\ M. W. Rhodes, P. Wichaisirimongkol, IST, Chiang Mai University, Chiangmai Thailand \\ H. Wiedemann, SLAC/SSRL, Menlo Park, California, USA
}

\begin{abstract}
Femtosecond (fs) electron and photon pulses become a tool of increasing importance to study dynamics in ultrafast processes. Such short electron pulses can be generated from a system consisting of a thermioniccathode RF-gun and a magnetic bunch compressor. The fs electron pulses can be used directly or used as a source to produce equally short electromagnetic radiation pulses via certain kind of radiation production processes. At the Fast Neutron Research Facility (FNRF), Thailand, we are especially interested in production of radiation in Farinfrared and X-ray regime. In the far-infrared wavelengths, the radiation emitted from fs electron pulses is emitted coherently resulting high intensity radiation. In the X-ray regime, development of fs X-ray sources is crucial for application in ultrafast sciene.
\end{abstract}

\section{INTRODUCTION}

Ultrashort electron and photon pulses, especially in the femtosecond (fs) time scale, have become important tools for study dynamics in ultrafast processes. Accesibility of such short pulses made it possible to monitor dynamics of chemical reactions, biomolecular processes and in timeresolved experiments with fs resolving power [1, 2]. The SURIYA project, to develop fs electron and photon sources, has been established at the Fast Neutron Research Facility (FNRF), Thailand, and is now under commissioning. A schematic layout of SURIYA set-up is shown in Fig. 1 consisting of an RF-gun with a thermionic cathode, an $\alpha$-magnet, a linear accelerator and beam transport components. The electron bunches of ultrashort duration, even at the low energy level without further acceleration, can be used directly for timeresolved analysis applications [1]. Furthermore, the electron bunches can be used to generate fs electro magnetic radiation pulses.

When accelerated, charged particles emit electro magnetic radiation and the radiation characteristics are different depending on radiation production mechanisms. Utilizing fs electron bunches to produce radiation with proper radiation production processes makes it possible to generate fs electromagnetic radiation pulses. Under SURIYA project, we are especially interested in production of far-infrared (FIR) radiation and X-ray. High intensity FIR radiation can be produced via coherent transition radiation (TR) while the X-ray can be produced by way of parametric $\mathrm{X}$-radiation (PXR).

\section{GENERATION OF FEMTOSECOND ELECTRON BUNCHES}

As shown in Fig. 1, fs electron bunches are generated from a thermionic-cathode and an $\alpha$-magnet serving as a magnetic bunch compressor. The specially-design RF-gun was studied and constructed [3]. It was designed such that the first particle in each S-band cycle will be exposed to maximum acceleration while any later particles will accumulate less energy. By this condition, higher energy particles will emerge first from the RF-gun followed by lower energy particles, consequently generating a welldefined correlation between energy and time. Electron bunches of 20-30 ps at 2-3 MeV from the RF-gun are then
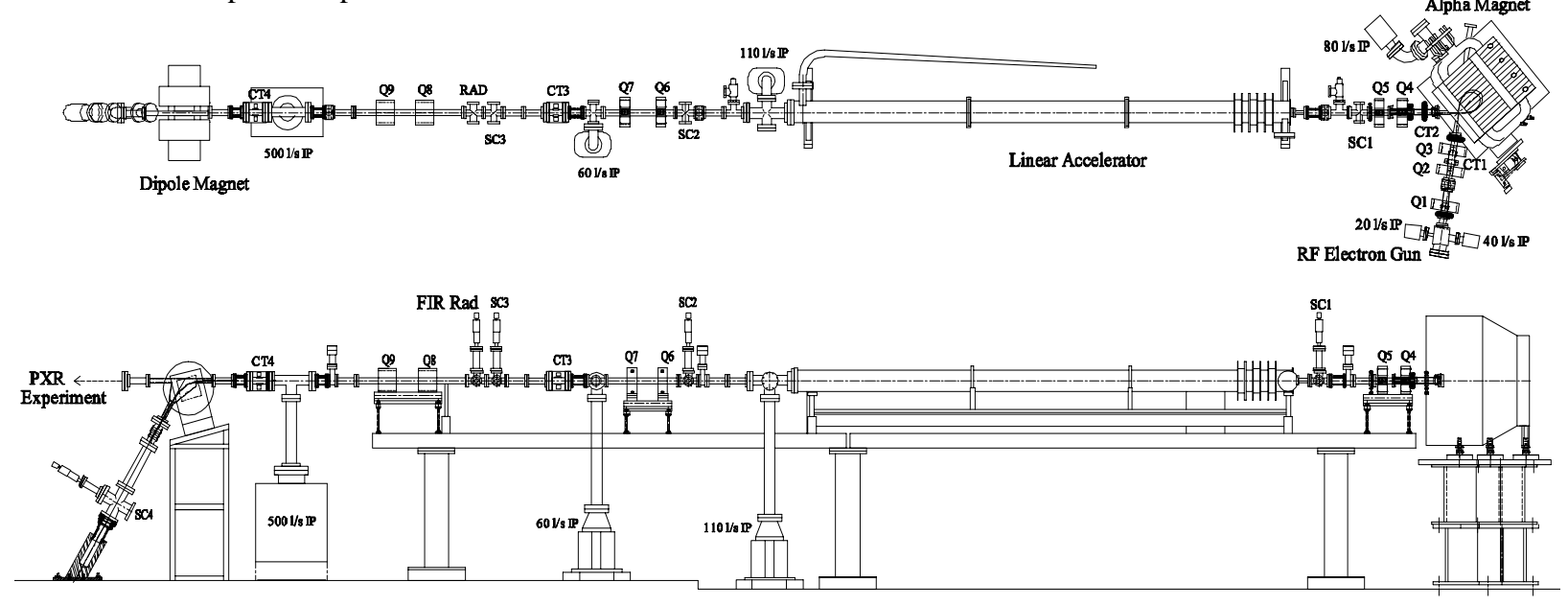

Figure 1: Schematic layout of the system at SURIYA of the Fast Neutron Research Facility.

*chlada@fnrf.science.cmu.ac.th 
compressed in an $\alpha$-magnet, where the particle path length increases with energy. This allows the lower energy particles, emitted later in each bunch, to catch up with the front for effective bunch compression. At the end of this process, the bunches are compressed to less than 1 ps. After acceleration in a single section S-band linear accelerator up to $20 \mathrm{MeV}$, the electrons are guided to experimental stations for radiation production.

\section{GENERATION OF COHERENT FAR-INFRARED RADIATION}

At a wavelength longer than the electron bunch length, the radiation is emitted coherently with the intensity scaling like $N^{2}$. With a typical number of electrons $N$ around $10^{8}$ to $10^{9}$ electrons in each bunch, the radiation intensity will be enhanced by the large factor. Intense FIR radiation can therefore be derived from relativistic fs electron bunches [4]. The total radiated power from a mono-energetic bunch can be written as $P(\omega)=P_{0}(\omega)[N+$ $N(N-1) f(\omega)]$, where $P_{0}(\omega)$ is the radiated power from a single electron. The form factor, $f(\omega)$, is the Fourier transform of the bunch distribution and the second term in the square bracket describes the coherent radiation. The factor $N f(\omega)$ will enhance the incoherent radiation intensity emitted by any radiation production processe.

Transition Radiation (TR) is emitted when a charged particle passes through an interface between two media with different dielectric constants. A thin Al-foil is employed to serve as the TR radiator which represents the transition between vacuum and conductor. A $45^{\circ}$-tilted Al-foil can be used as the TR radiator to produce backward TR emitted at $90^{\circ}$ with respect to the beam axis, as shown in Fig. 2.

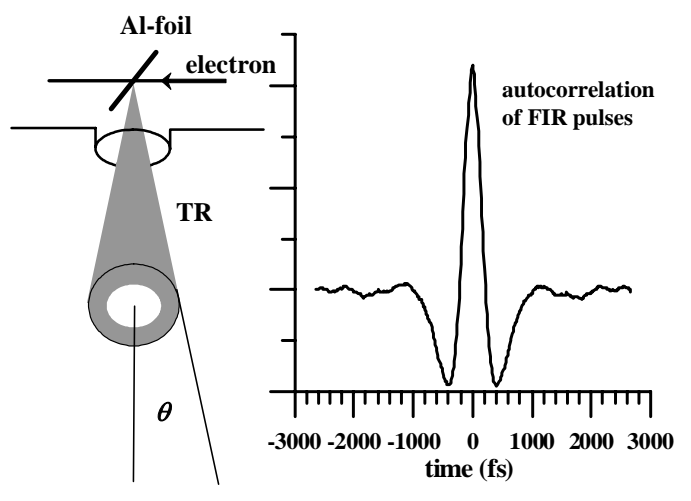

Figure 2: Coherent TR in the FIR from electron bunches.

The spectral-angular distribution of the emitted TR energy can be described by

$$
\frac{\mathrm{d}^{2} W}{\mathrm{~d} \omega \mathrm{d} \Omega}=\frac{r_{c} m_{c}^{2} \sin ^{2} \theta}{\pi^{2} c\left(1-\beta \sin ^{2} \theta\right)^{2}},
$$

where $\theta$ is the emission angle with respect to the electron beam axis. The intensity increases from zero in the forward direction to a broad peak at an angle $\theta=1 / \gamma$. Figure 2 also shows an autocorrelation of FIR pulses generated by approximately 120 fs of $26 \mathrm{MeV}$ electron beams from a similar system [4]. The autocorrelation pattern, which can be used to determine the radiation and electron pulse lengths, was taken by using a Michelson interferometer. As described by Eq. 1, the single electron TR spectrum is uniform up to a very high frequency, however, for coherent TR this uniform spectrum folds with the form factor of the electron bunch. Figure 3 shows a measured coherent TR spectrum obtained from the same measurement shown in Fig. 2. The radiation is broadband and its detectable spectrum reaches from microwaves to $120 \mathrm{~cm}^{-1}$ wavenumber. The FIR radiation brightness of coherent TR estimated for 50 fs electron bunches, expected in the near future at FNRF [3], extending to over $300 \mathrm{~cm}^{-1}$ wavenumber greatly exceeds that of a black body as well as that of synchrotron radiation as shown in Fig. 4.

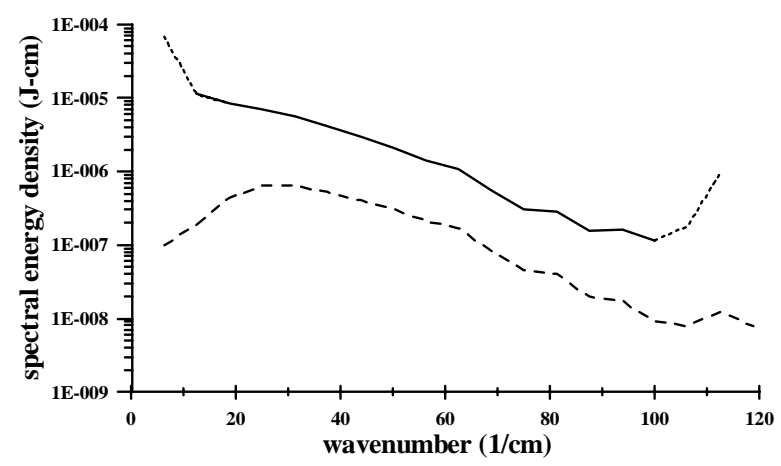

Figure 3: Coherent TR raw-spectrum (dashed-line) and the corrected spectrum (solid line) after applying the correction for beam splitter (BS) efficiency. The dottedsection occupy region near singularities of the BS efficiency.

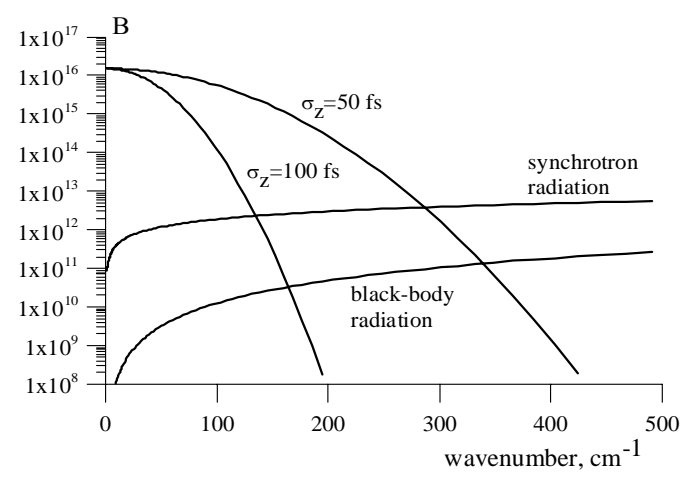

Figure 4: Radiation brightness B ( $\left.\mathrm{Ph} / \mathrm{s} / \mathrm{mm}^{2} / 100 \% \mathrm{BW}\right)$ of Coherent TR, blackbody and synchrotron radiation.

\section{GENERATION OF X-RAY PULSES}

Variety of radiation production mechanisms can be used to convert the fs electron bunches to x-ray pulses as for example bremsstrahlung, synchrotron-, channeling-, 
transition-, Smith-Purcell-, and parametric x-radiation as well as Compton scattering. Among those, the parametric $\mathrm{X}$-radiation (PXR) is suitable for $\mathrm{X}$-ray production using few tens $\mathrm{MeV}$ electron beams since the photon energy being produced is independent of the electron energy. The name PXR is accepted in most experimental publications for the X-ray emitted when a charged particle passing through a crystal [5-7]. There are two schemes for PXR generation from relativistic electrons: Laue and Bragg geometry. For Laue geometry, the radiation production is associated with crystallographic planes perpendicular to the slab surface while Bragg geometry deals with crystallographic planes oriented parallel to the slab surface. PXR is generated under the Bragg condition $d \sin \phi=n \lambda$ allowing us to select the photon energy by adjusting the crystal rotation angle $\phi$. The PXR production geometry can be described using Fig. 5 with a (100) silicon wafer at observation angle $\theta$.

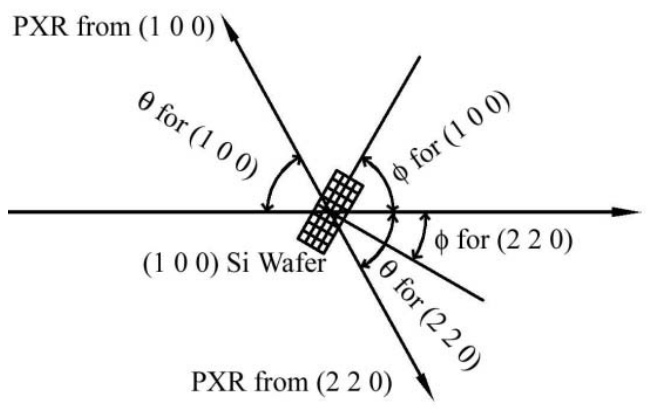

Figure 5: Geometry for PXR generation from a (100) silicon wafer.

The energy of PXR can be derived from [7]

$$
E=\bar{h} \omega=\frac{c \bar{h}|\vec{g} \cdot \vec{V}|}{c-\sqrt{\varepsilon} \vec{V} \cdot \vec{\Omega}}=\frac{\bar{h}|\vec{V}||\vec{g}| \sin \phi}{1-\beta \cos \theta}
$$

where $E=\mathrm{PXR}$ energy, $\omega=\mathrm{PXR}$ angular frequency, $c=$ velocity of light in vacuum, $\vec{g}=$ the reciprocal lattice vector, $\vec{V}=$ electron velocity, $\vec{\Omega}=$ direction of observation and $\varepsilon=$ dielectric constant of the crystal which becomes unity for X-rays. The reciprocal lattice magnitude in Eq. 2 can be calculated from Miller indicies of the plane $(h k l)$, and the lattice constant $a$ as $|\vec{g}|=(2 \pi / a) \sqrt{h^{2}+k^{2}+l^{2}}$. By comparing $|\vec{g}|$ for the (220)-plane and (100)-plane, one can see that the radiation associated with the (220)-plane results in a higher energy based on the higher magnitude of reciprocal lattice vector. Estimated PXR energy associated with the (220)-plane as a function of crystal rotation angle $\phi$ and observation angle $\theta$ by keeping $\theta=2 \phi$, as indicated by Bragg direction, are shown in Fig. 6.

Although, at very small observation angles, the PXR energy can be as high as $100 \mathrm{keV}$, the radiation is emitted in the direction close to the direction of the electron beams and makes it difficult to detect. PXR in 10-35 keV

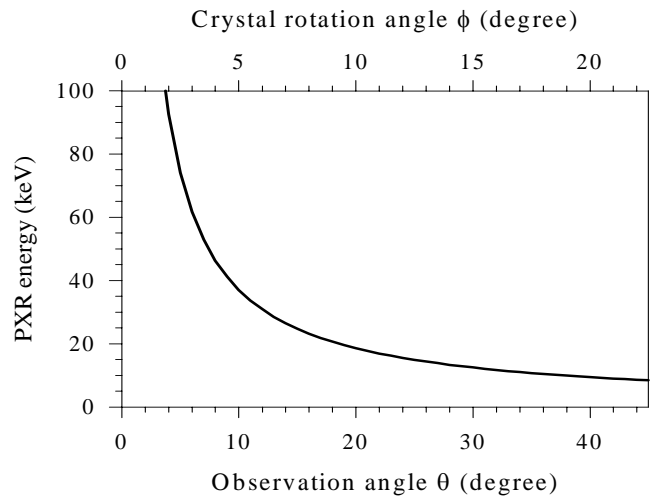

Figure 6: PXR energy from $\mathrm{Si}(220)$-plane as function of observation angle $\theta$ and crystal rotation angle $\phi$.

range can be obtained at more convenient observation angles of 10-45 degrees and thus become our main interest for X-ray pulses production.

\section{CONCLUSION}

With accessibility of fs electron bunches it is possible to generate high brightness, coherent, broadband FIR via TR and generate tunable $\mathrm{x}$-rays via PXR. The radiation pulses come in very short bursts of fs duration reflecting the electron bunch length and time structure. The high intensity fs FIR pulses can be applied for FIR spectroscopy allowing direct determination of complex refractive index [8]. The availability of fs X-rays will open up opportunities for time-resolved studies of ultrafast processes.

\section{ACKNOWLEDGEMENTS}

We would like to acknowledge the support of the Thailand Research Fund (TGR4580055), the National Research Council of Thailand, the Thai Royal Golden Jubilee Scholarship Program, the US Department of Energy, the HEPL of Stanford University, the Department of Physics, Faculty of Science, and Chiang Mai University.

\section{REFERENCES}

[1] H. Ihee et. al., Science, 291 (2001) 458.

[2] M. Bauer et. al., Phys. Rev. Lett., 87 (2001) 025501-1.

[3] S. Rimjaem et. al., NIM A, 533 (2004) 258.

[4] C. Settakorn, Generation and Use of Coherent Transition Radiation from Short Electron Bunches, Ph.D. thesis, Stanford University, California (2001).

[5] V. G. Baryshevsky and I. D. Feranchuk, NIM A, 228 (1985) 490.

[6] M.L. Ter-Mikaelian, High Energy Electromagnetic Process in Condensed Media. Wiley-Interscience, New York, 1972.

[7] A. V. Shchagin et. al., Phys. Lett. A, 148 (1990) 485.

[8] K. Wood et. al., Chem. Phys. Lett., 393 (2004) 159. 